\title{
Some Features of the Curvature of a Two-Dimensional Detonation Shock Front at a Simple Refraction Locus
}

\author{
P. Vidal, E. Bouton and H.-N. Presles \\ Laboratoire d'Energétique et de Détonique, URA 193 du CNRS, ENSMA, BP. 109, 86960 Futuroscope \\ cedex, France
}

\begin{abstract}
We present a theoretical study of the interaction of a constant-velocity two-dimensional detonation wave with its surrounding medium. For the case of pure refraction, we obtain exact expressions for the interface curvatures of the shock fronts in both the explosive (X) and its confinement (C) in terms of the detonation velocity $\mathrm{D}$, the material properties of $\mathrm{X}$ and $\mathrm{C}$ and, if the flow is cylindrically symmetric, the radius of the explosive charge. These relations are obtained from the constraints imposed on the flow derivatives of the pressure $P$ and the flow turning angle $\theta$ by the conservation laws, the boundary conditions at the curved shock fronts and the contact conditions matching $P$ and $\theta$ along the interface. This model is used in our numerical analysis of a polytropic explosive with a pressure-dependent decomposition rate and a polytropic confinement. We find that, for a given $\mathrm{D}$, the explosive's interface curvature $C_{x}$ decreases as the confinement's density increases.
\end{abstract}

\section{INTRODUCTION}

Detonation wave front dynamic in condensed explosives is currently studied by using evolution equations which relate the geometrical and kinematical properties of the wave surface. There are essentially two approaches. In the first one [1] the detonation is considered as a generalized CJ wave, specifically a curved partially reactive sonic hydrodynamic discontinuity. Under the assumption that the spatial and time flow derivatives are finite at the reactive side of this discontinuity, the analysis yields an hyperbolic evolution equation which relates the mean curvature, the normal velocity and the normal acceleration of the wave front surface. In the second one [2], the detonation is considered as a generalized ZND structure. Under the assumptions that the flow is quasi-one-dimensional and quasi-steady behind the leading shock, the analysis leads to a parabolic evolution equation which relates the mean curvature and the normal velocity of the detonation shock front surface. These evolution equations have the common feature to be solely dependent on the explosive material properties. To use these models for studying constant velocity curved twodimensional detonations, it is necessary to first define a relevant boundary condition at the edge of the explosive charge, precisely the detonation wave front slope as a function of its velocity. The shape and the velocity of the detonation wave front are then uniquely determined for a given transverse size of the explosive charge. 
In this short communication, attention is focused on a particular boundary condition for a constant velocity curved two-dimensional detonation shock front. Specifically, we study the case of a pure refraction which describe the case where the shock in the explosive (X) and the shock in the confinement $(\mathrm{C})$ of $\mathrm{X}$ match whithout any wave reflected in $\mathrm{X}$ or $\mathrm{C}$ [3]. Further, we shall restrict our analysis to the interaction point I of the shocks in $\mathrm{X}$ and $\mathrm{C}$ and we shall derive exact expressions for the curvatures of $\mathrm{OI}$ and $\mathrm{O} I$ at point $\mathrm{I}$. We then shall present the results of calculations performed with these expressions subjected to the assumptions that $\mathrm{X}$ and $\mathrm{C}$ are governed by polytropic equations of state and that the chemical energy release rate in $X$ obeys a pressure-dependent law $[4,5]$.

\section{ANALYSIS OF THE REFRACTION}

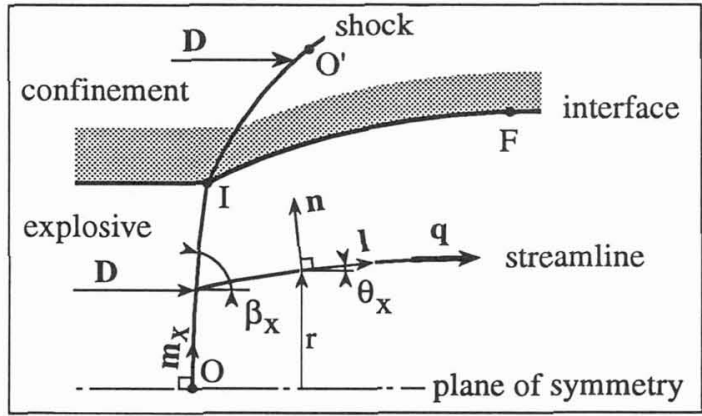

Figure 1. - Confined two-dimensional detonation

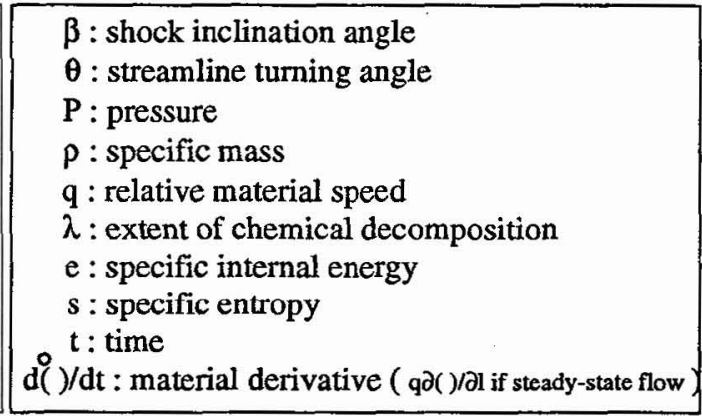

Table 1. - Nomenclature

Our study is based upon the two-dimensional (2D) Zeldovich-Von Neuman-Doring (ZND) model [6,7] : a 2D axisymmetric (2DA) or 2D plane (2DP) adiabatic inviscid reactive flow sustains a symmetrically curved shock propagating at constant velocity $\mathrm{D}$ in a quiescent explosive. In a SFF, the flows in $\mathrm{X}$ and $\mathrm{C}$ are steady-state in the vicinity of $\mathrm{OI}$ and O'I. We shall use the subscript $\mathrm{H}$ to denote a shocked state in $\mathrm{X}$ or $\mathrm{C}$ and use $\mathrm{x}$ and $\mathrm{c}$ as subscripts or superscripts to denote states in $\mathrm{X}$ and $\mathrm{C}$ respectively. We shall omit $\mathrm{x}$ and $c$ when referring to $X$ or $C$ indistinctively. Our other notations are given in Table 1. To describe such 2DP $(\alpha=0)$ or 2DA $(\alpha=1)$ flows in a SFF, we first write the balance equations for mass, momentum and energy in a system of curvilinear orthogonal coordinates defined by the streamlines and their normals as :

$$
\begin{array}{ll}
(\partial \operatorname{Ln}(\rho) / \partial \mathrm{l})+(\partial \operatorname{Ln}(\mathrm{q}) / \partial \mathrm{l})+(\partial \theta / \partial \mathrm{n}) & =-\alpha \sin (\theta) / \mathrm{r} \\
(\partial \operatorname{Ln}(\mathrm{q}) / \partial \mathrm{l})+(\partial \mathrm{P} / \partial \mathrm{l}) /\left(\rho \mathrm{q}^{2}\right) & =0 \\
(\partial \theta / \partial \mathrm{l})+(\partial \mathrm{P} / \partial \mathrm{n}) /\left(\rho \mathrm{q}^{2}\right) & =0 \\
(-\partial \operatorname{Ln}(\rho) / \partial \mathrm{l})+(\partial \mathrm{P} / \partial \mathrm{l}) /\left(\rho \mathrm{c}^{2}\right) & =\sigma \cdot \mathrm{w} / \mathrm{q}
\end{array}
$$

The (frozen) sound speed $c$ and the thermicity vector $\sigma$ can be expressed as functions of $P, \rho$ and $\lambda$ by using a specified $e(P, \rho, \lambda)$ equation of state. The decomposition rate vector $w$ is also to be considered as depending on $P, \rho$ and $\lambda$. In nonreactive media, $\lambda, \sigma$ and $w$ are equal to zero. 


$$
\begin{aligned}
& \mathrm{c}^{2}=(\partial \mathrm{P} / \partial \rho) \lambda, s=\left(\mathrm{P}^{-2}-(\partial \mathrm{e} / \partial \rho) \lambda, \mathrm{P}\right) /(\partial \mathrm{e} / \partial \mathrm{P}) \lambda, \rho \\
& \rho \mathrm{c}^{2} \sigma=(\partial \mathrm{P} / \partial \lambda)_{\mathrm{e}, \rho} \quad ; \quad\left(\mathrm{d}^{0} \lambda / \mathrm{dt}\right)=w(\mathrm{P}, \rho, \lambda)
\end{aligned}
$$

We next recall [8] that the shock values of any dependent variable $g=(\rho, q, P, \theta)$ can be expressed as a function of $\beta$ and $D$ by using the jump relations for an oblique shock and the equation of state of the medium under consideration. $\lambda_{\mathbf{H}}$ is equal to zero (ZND model). The shock values of the partial derivatives of the latter variables thus satisfy the following geometrical identity (Figure 1) :

$$
\cos \left(\beta-\theta_{H}\right)(\partial g / \partial)_{H}+\sin \left(\beta-\theta_{H}\right)(\partial g / \partial n)_{H}=\left(d_{H_{H}} / d \beta\right) C
$$

where $C=(\mathrm{d} \beta / \mathrm{dm})=\sin (\beta)\left(\mathrm{d} \beta / \mathrm{dr}_{\mathrm{H}}\right)$ denotes the curvature of a meridian shock line. Applying the identity (5) to the variables $P$ and $\theta$ yields two relations which, together with the relations (1) to (4), defines a linear nonhomogeneous system of six equations in the shock values of the six partial derivatives involved in it. Upon resolution, we obtain in particular

$$
\begin{aligned}
& (\partial \mathrm{P} / \partial \mathrm{l})_{\mathrm{H}}=\mathrm{A}_{\mathrm{p}} \mathrm{C}-\mathrm{B}_{\mathrm{p}} \\
& (\partial \theta / \partial 1)_{\mathrm{H}}=\mathrm{A}_{\theta} C-\mathrm{B}_{\theta} \\
& \left(\rho \mathrm{q}^{2}\right)_{\mathrm{H}}^{-1} \Delta \mathrm{A}_{\mathrm{p}}=\cos \left(\beta-\theta_{\mathrm{H}}\right)\left(d \mathrm{P}_{\mathrm{H}} / \mathrm{d} \beta\right) /\left(\rho_{\mathrm{H}} \mathrm{q}_{\mathrm{H}}^{2}\right)+\sin \left(\beta-\theta_{\mathrm{H}}\right)\left(\mathrm{d} \theta_{\mathrm{H}} / \mathrm{d} \beta\right) \\
& (\rho q)_{\mathrm{H}}^{-1} \Delta \mathrm{B}_{\mathrm{p}}=\sin ^{2}\left(\beta-\theta_{\mathrm{H}}\right)\left\{\sigma_{\mathrm{H}} \mathrm{w}_{\mathrm{H}} / \mathrm{q}_{\mathrm{H}}-\alpha \sin \left(\theta_{\mathrm{H}}\right) / \mathrm{r}_{\mathrm{H}}\right\} \\
& \Delta \mathrm{A}_{\theta}=-\left(1-\mathrm{M}_{\mathrm{H}}^{2}\right) \sin \left(\beta-\theta_{\mathrm{H}}\right)\left(\mathrm{dP} \mathrm{P}_{\mathrm{H}} / \mathrm{d} \beta\right) /\left(\rho_{H} q_{H}^{2}\right)+\cos \left(\beta-\theta_{\mathrm{H}}\right)\left(\mathrm{d} \theta_{\mathrm{H}} / \mathrm{d} \beta\right) \\
& \Delta \mathrm{B}_{\theta}=\cos \left(\beta-\theta_{\mathrm{H}}\right) \sin \left(\beta-\theta_{\mathrm{H}}\right)\left(\sigma_{\mathrm{H}} \mathrm{w}_{\mathrm{H}} / \mathrm{q}_{\mathrm{H}}-\alpha \sin \left(\theta_{\mathrm{H}}\right) / \mathrm{r}_{\mathrm{H}}\right\} \\
& \Delta=1-\mathrm{M}_{\mathrm{H}}^{2} \sin ^{2}\left(\beta-\theta_{\mathrm{H}}\right) \quad ; \quad \mathrm{M}=\mathrm{q} / \mathrm{c}
\end{aligned}
$$

The coefficients $A_{p}, A_{\theta}, B_{p}, B_{\theta}$ and $\Delta$ are function of $\beta$ and $D$. The coefficients $B_{p}$ and $B_{\theta}$ also depend on $r_{H}$ for a 2DA explosive charge.

We finally write the contact conditions for $\mathrm{X}$ and $\mathrm{C}$ by matching the pressures and the flow directions along the interface IF as :

$$
\begin{aligned}
& \mathrm{P}_{\mathrm{X}}=\mathrm{P}_{\mathrm{c}} \\
& \theta_{\mathrm{X}}=\theta_{\mathrm{c}} \\
& (\partial \mathrm{P} / \partial \mathrm{l})_{\mathrm{X}}=(\partial \mathrm{P} / \partial \mathrm{l})_{\mathrm{c}} \\
& (\partial \theta / \partial \mathrm{l})_{\mathrm{X}}=(\partial \theta / \partial \mathrm{l})_{\mathrm{c}}
\end{aligned}
$$


These matching conditions apply along the interface IF. It is important to acknowledge that they also apply at the intersection point I of shocks OI and O'I when there is no reflected wave in $\mathrm{X}$ or $\mathrm{C}$ as for a pure refraction. The shock relations (6) and (7) can thus be used to evaluate the derivatives in relations (10) and (11) at point $I$. This operation yields a linear nonhomogeneous system of two equations in the two interface values, $C_{X}$ and $C_{c}$, of the curvatures of shocks OI and O'I. We obtain

$$
\begin{aligned}
& C_{x}=\left\{\left(B_{\theta}^{x}-B_{\theta}^{c}\right) A_{p}^{c}-\left(B_{p}^{x}-B_{p}^{c}\right) A_{\theta}^{c}\right\} / \Delta \\
& C_{c}=\left\{\left(B_{\theta}^{x}-B_{\theta}^{c}\right) A_{p}^{x}-\left(B_{p}^{x}-B_{p}^{c}\right) A_{\theta}^{x}\right\} / \Delta \\
& \Delta=A_{\theta}^{x} A_{p}^{c}-A_{p}^{x} A_{\theta}^{c}
\end{aligned}
$$

The conditions (8) and (9) define the values of the shock angles $\beta_{x}$ and $\beta_{c}$ at $I$ as functions of the velocity $\mathrm{D}$ and the material shock properties of $\mathrm{X}$ and $\mathrm{C}$. The interface values of the curvatures of OI and $\mathrm{O}^{\prime} \mathrm{I}$ consequently depend on $\mathrm{D}$, the material shock properties of $\mathrm{X}$ and $\mathrm{C}$, the chemical decomposition rates and, for a 2DA explosive charge, on the radius of the explosive charge.

\section{RESUlTS OF A MODEL CALCULATION}

We have specialized the above results to the case of a 2DP explosive charge $(\alpha=0)$ bounded with a nonreactive confinement $\left(\lambda_{\mathrm{c}}=0, \sigma_{\mathrm{c}}=0, \mathrm{w}_{\mathrm{c}}=0\right)$ and have introduced in our analysis the following simplifying assumptions (the subscript 0 will denote the initial conditions) :

1- The inital pressure $P_{0}$ can be neglected with respect to the shock pressure $P_{H}$. In condensed explosives, typical $\mathrm{P}_{0} / \mathrm{P}_{\mathrm{H}}$ ratios are $10^{-5}$.

2- A global irreversible reaction can model the exothermic process in the reaction zone of $\mathrm{X}$ :

$$
\text { Explosive } \rightarrow \text { Products }
$$

Such a reaction is accordingly described by a single scalar variable $\lambda_{x}$ that represents the mass fraction of burnt explosive and specifies the fraction of heat released per unit mass of $\mathrm{X}$.

3- The material properties of $\mathrm{X}$ and $\mathrm{C}$ are governed by polytropic equations of state :

$$
e(P, \rho, \lambda)=e_{0}-\lambda Q+P / \rho(\gamma-1)
$$

where $\gamma$ and $Q$ denote the polytropic index and the total amount of heat avalaible per unit mass respectively. Here, $Q_{c}$ is equal to zero since we have chosen a nonreactive confinement.

4- The global chemical decomposition rate of $\mathrm{X}$ obeys a pressure-dependent law in the form :

$$
\mathrm{w}_{\mathbf{X}}=\tau^{-1}\left(\mathrm{P}_{\mathbf{X}} / \mathrm{P}_{\mathrm{ref}}\right)^{\mathrm{a}}\left(1-\lambda_{\mathrm{X}}\right)^{\mathrm{b}}
$$


where $\tau$ is a characteristic chemical time, $P_{\text {ref }}$ is a characteristic pressure and $a$ and $b$ are nondimensional constants.

Assumptions 1, 2 and 3 lead to the following one-dimensional SS-DW velocity [6,7]:

$$
D^{*}=\sqrt{2\left(\gamma_{x}^{2}-1\right) Q_{x}}
$$

The velocity $D^{*}$, the initial specific mass $\rho_{0 x}$ and the characteristic time $\tau$ were used to nondimensionalize the other variables and, without loss in generality, the characteristic pressure $P_{\text {ref }}$ was set equal to $\rho_{0 x} D^{* 2}$.

We next used formula (12) to determine how the relationship between the explosive's interface-curvature $\mathrm{C}_{\mathrm{X}}$ and the DW velocity $\mathrm{D}$ depends on the properties of $\mathrm{C}$ and on the parameter a of the decomposition rate (15). The control parameters of our calculations are the nondimensional velocity $\mathrm{D} / \mathrm{D}^{*}$, the polytropic indices $\gamma_{\mathrm{X}}$ and $\gamma_{\mathrm{c}}$, the relative density $\rho_{0 \mathrm{c}} / \rho_{0 \mathrm{X}}$, and the parameter a of the decomposition rate. The values of $D^{*}, \rho_{0 \mathrm{x}}, \rho_{0 \mathrm{c}}, \gamma_{\mathrm{X}}$ and $\gamma_{\mathrm{c}}$ were chosen to model the liquid explosive nitromethane $\left(D^{*}=0.63 \mathrm{~cm} / \mu \mathrm{s}, \rho_{0 \mathrm{x}}=\right.$ $\left.1.128 \mathrm{gr} / \mathrm{cm}^{3}, \gamma_{\mathrm{x}}=2.4\right)$ confined in steel $\left.\left(\rho_{0 \mathrm{c}} / \rho_{0 \mathrm{x}}=7, \gamma_{\mathrm{c}}=2.75\right)\right)$ or titanium $\left(\rho_{0 \mathrm{c}} / \rho_{0 \mathrm{x}}=4, \gamma_{\mathrm{c}}=1.54\right)$ tubes $[9,10]$. These materials meet the pure refraction conditions in the range of the selected values for $D / D^{*}(0.9-1.0)$. For polytropic materials, the values of the angles $\beta_{\mathrm{X}}$ and $\beta_{\mathrm{c}}$ are independent of $D$. Here we found:

$$
\begin{aligned}
& \beta_{x}=81.15 \text { degrees and } \beta_{c}=23.21 \text { degrees for } \rho_{0 d} / \rho_{0 x}=7 \\
& \beta_{x}=73.87 \text { degrees and } \beta_{c}=24.43 \text { degrees for } \rho_{0 d} / \rho_{0 x}=4
\end{aligned}
$$

Figure 2 presents the qualitative trend of our $\mathrm{D}-\mathrm{C}_{\mathrm{X}}$ relation for polytropic materials. One observes that, for a given velocity $\mathrm{D}$, the higher the density of the confinement, the smaller the explosive's interface curvature Our numerical results are summarized in Figure 3 which plots the nondimensionalized wave velocity $D / D^{*}$ versus the nondimensionalized interface curvature $\tau D * C_{x}$. The curves exhibits the following trends:

1- For a given velocity $D$, the absolute value of the explosive's interface-shock-curvature, $-C_{X}$, decreases as the confinement's density increases. Also, the distance between curves associated with two confinements and the same value of the parameter a decreases as the value of a increases.

2- Depending on the pressure exponent in the decomposition rate, the slope of the curves can be positive infinite or negative.

3- The same considerations apply to the dependence of the normal velocity $D \sin \left(\beta_{X}\right)$ and the explosive's interface curvature. 


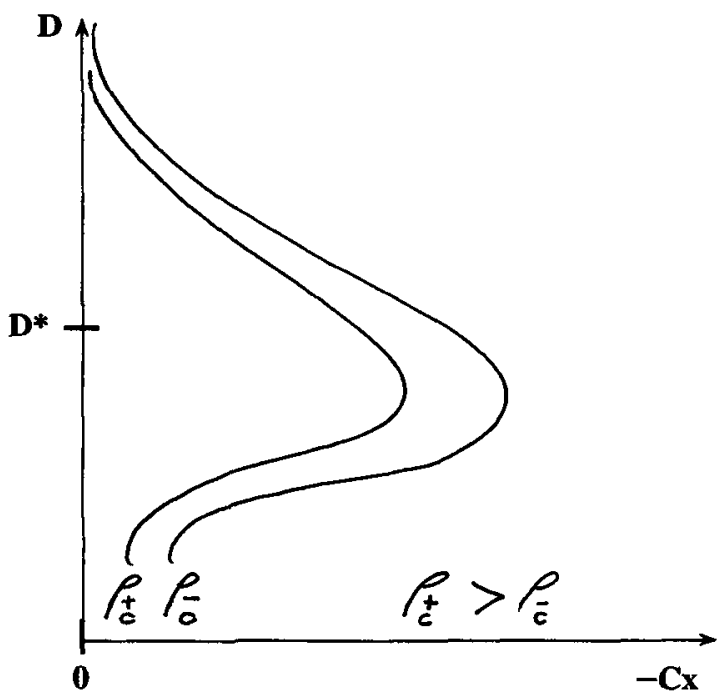

Figure 2. - Qualitative trends of the D-Cx relationship in the case of a polytropic explosive and two polytropic confinements.

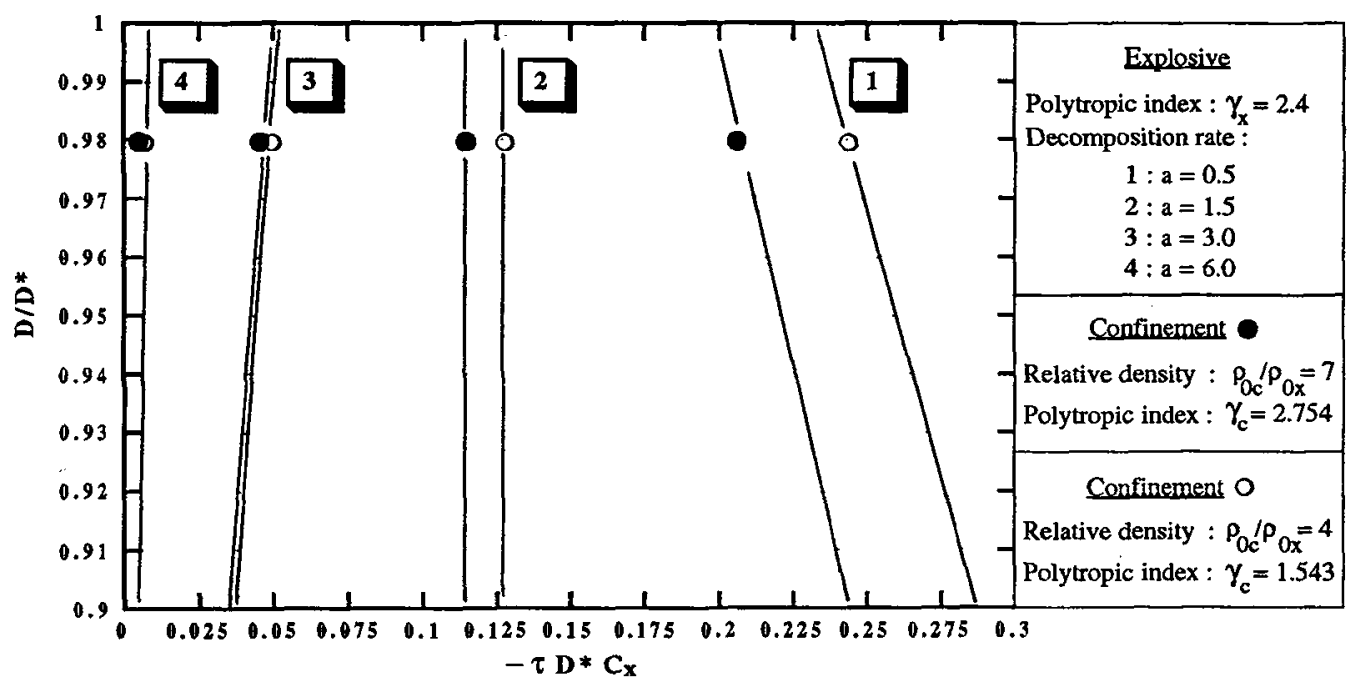

Figure 3. -Plots of the nondimensionalized detonation velocity $\mathrm{D} / \mathrm{D}^{*}$ versus the nondimensionalized explosive's interface curvature $-\tau D^{*} C_{\mathrm{x}}$ for a polytropic explosive, two polytropic confinements and the decomposition rate law $w_{x}=\tau^{-1}\left(p_{x} / p_{\text {ref }}\right)^{a}\left(1-\lambda_{x}\right)^{v}$ (two-dimensional plane charge). 


\section{CONCLUSIONS}

Current modelling of self-sustaining curved two-dimensional detonation shock fronts is based upon a compatibility relationship between the normal velocity (Dn) and the curvature (C) of the detonation shock front that only depends on the properties of the explosive [2,11]. This relationship is obtained by integrating the flow equations between the leading shock and the rear boundary of its domain of dependence (the "sonic locus") under the assumption that the flow which sustain the shock is quasi onedimensional and quasi steady. Our analysis indicates that there exists a situation where the geometrical characteristics of a curved detonation wave front can be obtained in closed forms from straight geometrical considerations without approximating the flows behind the curved shock fronts and without restricting the forms of the equations of state and the chemical decomposition rates. It also indicates that, in this situation, these characteristics depend on both the explosive and the confinement material properties. Our formula (12)for the explosive's interface shock curvature can thus be used to assess the accuracy of the (Dn-C) relationship, in the close vicinity of the interaction point and to calibrate shock equations of state and decomposition rates in condensed explosives from velocity measurements and shock-front shape records of confined two-dimensional self-sustaining detonation waves.

Finally, we suggest that one should distinguish between two cases of dynamical behavior for detonation shock fronts propagating in two-dimensional charges under the pure refraction boundary condition. In the first case, the explosive has sufficiently short a reaction zone so that only a very narrow domain in the vicinity of the explosive-confinement interface has a significant influence on the detonation shock front geometry. The dynamical behavior of such explosives would consequently be correctly described by evolution equations uniquely determined by the properties of the explosive. In the second case, the explosive has sufficiently long a reaction zone so that a wide domain of the flow in the explosive receives informations from the explosive-confinement interface. In this case, it seems reasonable to expect that the dependence of the detonation shock front geometry on the confinement material properties evidenced in our work is not restricted to the close vicinity of the interaction point but extends to a large part of the detonation shock front and, presumably, even on the explosive charge axis. Consequently, the dynamical behavior of the detonation shock front would here obey more complicated evolution equations than in the first case.

\section{REFERENCES}

[1] Brun L., rapport CEA-DAM, CEV-DPM, nº DO879023, (1989).

[2] Klein R., SIAM J.Appl.Math., 53, 5, (1993) 1401-1435.

[3] Stewart D.S. and Bdzil J.B., "Examples of detonation shock dynamics for detonation wave spread applications", Ninth Symposium (International) on Detonation, OCNR 113291-7,1, (1989) 773-783.(See the discussion by Bdzil at the end of the paper). 
[4] Vidal P., Cowperthwaite M., Presles H.-N., Fontaine D., C.R.Acad.Sci.Paris, t.315, Série II, (1992) 791-794.

[5] Vidal P., Cowperthwaite M., Presles H.-N., Fontaine D., C.R.Acad.Sci.Paris, t.316, Série II, (1993) $177-180$.

[6] Zeldovich Ya.B., Kompaneets S.A., Theory of detonation, (Academic Press, New York, 1960).

[7] Fickett W., Davis W.C., Detonation, (University of California Press, Berkeley,1979).

[8] Courant R., Friedrichs K., Supersonic flow and shock waves, (Intersciences, 1948).

[9] Marsh S.P., LASL Shock Hugoniot Data, (University of California Press, Berkeley, 1980.

[10] Presles H.-N. , Contribution à l'étude de la détonation de mélanges liquides binaires à base de nitrométhane, (Thèse de doctorat ès sciences physiques, Université de Poitiers, 1979).

[11] Stewart D.S., Bdzil J.B., Combustion and flame, 72, (1988) 311-323. 